EPJ Web of Conferences 66, 04001 (2014)

DOI: $10.1051 /$ epjconf/20146604001

(C) Owned by the authors, published by EDP Sciences, 2014

\title{
Quarkonium production in heavy-ion collisions
}

\author{
Roberta Arnaldi ${ }^{1, a}$ \\ ${ }^{1}$ Istituto Nazionale di Fisica Nucleare, Sezione di Torino, Via P. Giuria 1, I-10125 Torino, Italy
}

\begin{abstract}
The production of quarkonium states plays a crucial role among the probes to investigate the formation of the plasma of quarks and gluons (QGP) in heavy-ion collisions. A review of the charmonium and bottomonium production, mainly focussing on the latest results from the LHC experiments, is presented.
\end{abstract}

\section{Introduction}

Quarkonia ( $c \bar{c}$ or $b \bar{b}$ bound states) are considered important probes of the strongly interacting medium, the so-called quark gluon plasma (QGP), created in heavy-ion collisions. In a hot and deconfined medium, in fact, quarkonium production is expected to be significantly suppressed with respect to the pp yield scaled by the number of binary nucleon-nucleon collisions. The origin of such a suppression, which sets in in the QGP, is the color screening of the force which binds the $c \bar{c}(b \bar{b})$ state [1]. In this scenario, quarkonium suppression should occur sequentially, according to the binding energy of each meson: strongly bound states, as the $\mathrm{J} / \psi$ and $\Upsilon(1 \mathrm{~S})$, should melt at higher temperatures with respect to the more loosely bound ones, as the $\psi(2 \mathrm{~S})$ and $\chi_{c}$, for the charmonium family, or the $\Upsilon(2 S)$ and $\Upsilon(3 S)$ for the bottomonium one. As a consequence, the in-medium dissociation probability of such states should provide an estimate of the initial temperature reached in the collisions [2]. However, the prediction of the suppression pattern is complicated by several factors as the feed-down contributions from higher-mass resonances into the observed quarkonium yield, the B-hadrons decay into charmonium, or other competing mechanisms, as quarkonium production via (re)combination and cold nuclear matter effects.

Increasing, in fact, the center of mass energy of the collisions $(\sqrt{s})$, an increase of the production of $c$ and $\bar{c}$ quarks is expected. Therefore, in high $\sqrt{s}$ collisions, the abundance of $c$ and $\bar{c}$ quarks might lead to a new source of charmonia generation, due to the (re)combination of these quarks during the collision history [3] or at the hadronization [4, 31]. This new additional source of charmonium production should contribute by enhancing the $\mathrm{J} / \psi$ yields.

The in-medium modification of quarkonium production is usually quantified through the nuclear modification factor $R_{\mathrm{AA}}$ defined as the ratio of quarkonium yields in A-A collisions and the expected value obtained scaling the pp yield by the average number of nucleon-nucleon collisions $\left(\left\langle N_{\text {coll }}\right\rangle\right)$, obtained from a Glauber model calculation [6]:

$$
R_{\mathrm{AA}}=\frac{\text { Yield }_{\mathrm{AA}}^{q \bar{q}}}{\left\langle N_{\mathrm{coll}}\right\rangle \times \text { Yield }_{\mathrm{pp}}^{q \bar{q}}}
$$

a. e-mail: arnaldi@to.infn.it

This is an Open Access article distributed under the terms of the Creative Commons Attribution License 2.0, which permits unrestricted use, distribution, and reproduction in any medium, provided the original work is properly cited. 
$R_{\mathrm{AA}}=1$ means that the quarkonium yield in A-A scales with the number of nucleon-nucleon collisions, while $R_{\mathrm{AA}} \neq 1$ implies that the quarkonium is affected by the medium (in particular, if $R_{\mathrm{AA}}<1$ the quarkonium yield is suppressed, if $R_{\mathrm{AA}}>1$ it is enhanced).

It is worth noting that the binary scaling can be broken not only because of the presence of a hot medium, but also because of cold nuclear matter effects (CNM). For example, the kinematic distributions of partons in nuclei are different from those in free protons and neutrons (nuclear shadowing [7-10]), affecting, in this way, the production cross section of the $q \bar{q}$ pair. In a similar way, according to Color-Glass Condensate effective theory [11, 12], a gluon saturation is also expected to affect quarkonium production. Furthermore parton energy loss may decrease the pair momentum $[13,14]$, causing a reduction of the quarkonium production at large longitudinal momentum. Finally, while the $q \bar{q}$ pair evolves towards the final quarkonium state, it may also interact with the medium and eventually break-up. Hence, for the interpretation of A-A results, data from p-A collisions are crucial because they allow us to disentangle these cold nuclear matter effects from those related to the formation of a hot QCD medium.

Studies performed in the last twenty-five years at the SPS $\left(\sqrt{s_{N N}}=17 \mathrm{GeV}\right)$ and RHIC facilities $\left(\sqrt{s_{N N}}=39-200 \mathrm{GeV}\right)$ have, indeed, shown a reduction of the $\mathrm{J} / \psi$ production yield beyond the expectations due to the cold nuclear matter effects (as nuclear shadowing and $c \bar{c}$ break-up) [15-18]. In spite of the very different center of mass energy, the amount of suppression observed by SPS and RHIC experiments is similar [19]. Furthermore, unexpectedly, at RHIC a stronger $\mathrm{J} / \psi$ suppression has been measured at forward with respect to mid-rapidity. These observations suggest the existence of the aforementioned additional $\mathrm{J} / \psi$ production mechanism, which start to set in already at RHIC energies. This mechanism, based on the combination of initially uncorrelated $c$ and $\bar{c}$ pairs can counteract the quarkonium suppression in the QGP.

Therefore, the measurement of charmonium production is especially promising at the LHC, where the high-energy density of the medium and the large number of $c \bar{c}$ pairs produced in central $\mathrm{Pb}-\mathrm{Pb}$ collisions should help to disentangle suppression and (re)combination scenarios. Furthermore, at the LHC energies also bottomonium states, which were barely accessible at lower energies, are now abundantely produced. Bottomonium resonances should help sheding some light on the processes affecting the quarkonium behaviour in the hot matter. Upsilons are, in fact, expected to be less affected by production through (re)combination processes, due to the much smaller abundance of $b$ and $\bar{b}$ quarks in the medium with respect to $c$ and $\bar{c}$. Also cold nuclear matter effects, as shadowing, are expected to be less important for bottomonia than for charmonia states [20].

\section{Quarkonium production at LHC energies}

All the LHC experiments (ALICE, ATLAS, CMS, LHCb) have carried out studies on quarkonium production either in $\mathrm{Pb}-\mathrm{Pb}$ collisions or in $\mathrm{p}-\mathrm{A}$ collisions, together with the study of pp interactions (not covered in this proceeding). The different kinematic coverage of the four experiments allow us to have complementary quarkonium production results spanning $\sim 4$ rapidity $(y)$ units, from 0 to high transverse momentum $\left(p_{\mathrm{T}}\right)$. ATLAS [21] and CMS [22, 23] measure quarkonium production reconstructing charmonium and bottomonium states in the dimuon decay channel. They both cover the mid-rapidity region (depending on the quarkonium state under study and on the $p_{\mathrm{T}}$ range investigated, the CMS rapidity coverage can reach $|y|<2.4$, and a similar $y$ range is also covered by ATLAS). ALICE $[24,25]$ is designed to study quarkonium in two rapidity zones: at mid-rapidity $(|y|<0.9)$ in the dielectron decay channel and at forward rapidity $(2.5<y<4)$ in the dimuon channel, in both cases down to 0 transverse momentum. LHCb has taken part only to the p-A LHC program and results on quarkonium production, reconstructed through the dimuon decay channel, are provided at forward $y$ $(2<y<4.5)[26]$. 
The inclusive (prompt $\mathrm{J} / \psi$ plus those coming from B-hadron decays) $\mathrm{J} / \psi$ nuclear modification factor is studied by ALICE as a function of the centrality of the collisions $\left(\mathrm{N}_{\text {part }}\right)$ [25]. The $\mathrm{J} / \psi$ cross section measured in pp collisions at $\sqrt{s}=2.76 \mathrm{TeV}$ is used as reference for the $R_{\mathrm{AA}}$ evaluation [27]. In Fig. 1 the $R_{\mathrm{AA}}$ centrality dependence is compared with the results of the PHENIX experiment. At lower energy there is an increasing suppression moving towards central collisions, while ALICE result clearly indicates a saturation both at forward (left) and at mid-rapidity (right). In both $y$ ranges there is a clear evidence for a smaller suppression at LHC with respect to RHIC energy, but no final conclusion can be drawn on the relative size of the suppression measured in ALICE at forward or mid-rapidity, mainly due to the large normalization error corresponding to the uncertainty associated to the reference pp cross section. Partonic transport models which include a (re)generation process for $\mathrm{J} / \psi$ due to the recombination of $c \bar{c}$ pairs along the history of the collisions indeed predict such a behaviour [28-30], the smaller suppression at the LHC being due to the larger $c \bar{c}$ pair multiplicity which compensates the suppression from color screening in the deconfined phase. A similar behaviour is obtained by the statistical model [31], where the $\mathrm{J} / \psi$ yield is completely determined by the chemical freeze-out conditions and by the abundance of $c \bar{c}$ pairs.
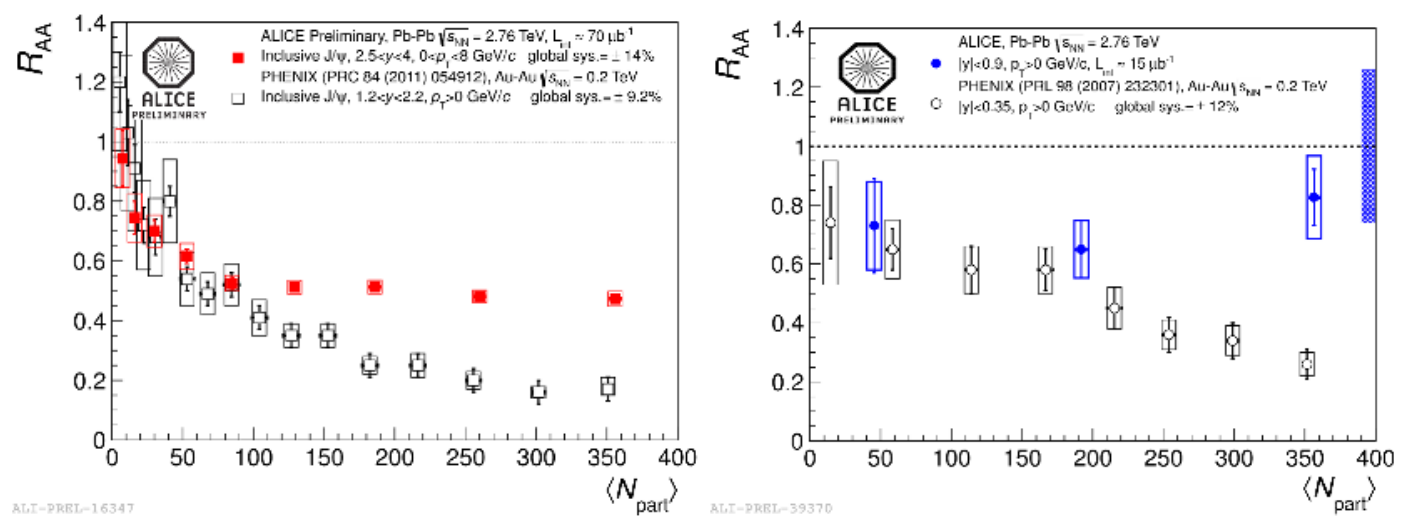

Figure 1. ALICE [25] (closed symbols) and PHENIX [17, 18] (open symbols) inclusive J/ $\psi$ nuclear modification factor versus centrality at forward rapidity (left) and at mid-rapidity (right)

The (re)combination process is expected to be dominant in central collisions and, for kinematical reasons, it should contribute mainly at low $p_{\mathrm{T}}$, while it becomes negligible as the $\mathrm{J} / \psi p_{\mathrm{T}}$ increases. This behaviour can be investigated by further studying the $R_{\mathrm{AA}} p_{\mathrm{T}}$ dependence in centrality bins, as shown in Fig. 2 (left) for $J / \psi$ detected in the forward rapidity region. While in the peripheral bin (40-90\%) the $p_{\mathrm{T}}$ dependence is negligible, in the most central bin (0-20\%) the suppression increases by $60 \%$ moving towards high $p_{\mathrm{T}}$. Models including a $p_{\mathrm{T}}$-dependent contribution from (re)combination which amounts, at low $p_{\mathrm{T}}$, to $60 \%$ in central and $30 \%$ in peripheral collisions [28, 29], provide a reasonable description of the data. This observation is confirmed also by CMS J/ $\psi$ results $[22,23]$. CMS measures high $p_{\mathrm{T}}$ $\mathrm{J} / \psi\left(p_{\mathrm{T}}>6 \mathrm{GeV} / c\right)$, i.e. $\mathrm{J} / \psi$ in a kinematic region where (re)combination should play a negligible role. Contrarily to what is observed by ALICE, the $\mathrm{J} / \psi$ yield is indeed increasingly suppressed towards the most central collisions, but it does not show a significant dependence on $p_{\mathrm{T}}$, in the high $p_{\mathrm{T}}$ range under study, as shown in Fig. 2 (right). The differential $R_{\mathrm{AA}}$ results previously discussed can be considered as strong hints for an important contribution of (re)combined $\mathrm{J} / \psi$ at low $p_{\mathrm{T}}$, setting in at the LHC. An independent confirmation of this hypothesis comes from the study of the elliptic flow $\left(\mathrm{v}_{2}\right)$ of $\mathrm{J} / \psi$. If (re)combination effects are sizeable, then the corresponding $\mathrm{J} / \psi$ would inherit the flow related to 

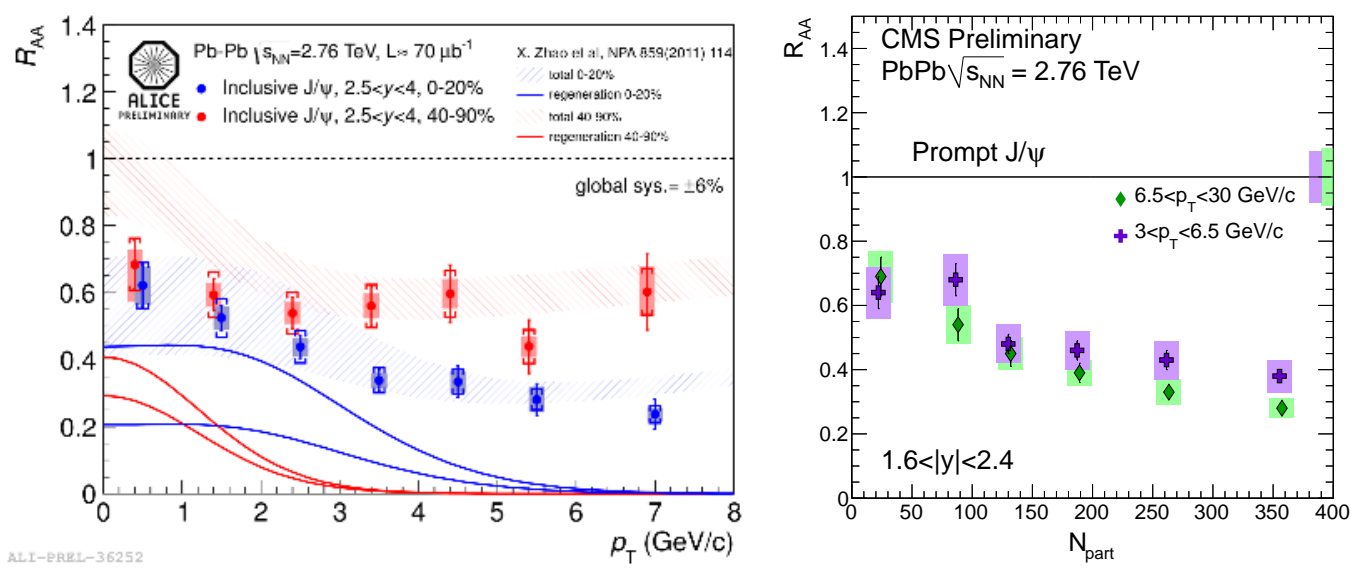

Figure 2. ALICE inclusive $\mathrm{J} / \psi R_{\mathrm{AA}}$ versus $p_{\mathrm{T}}$ [25], in two centrality classes (0-20\% and 40-90\%), compared to theoretical calculations (left). CMS prompt $\mathrm{J} / \psi R_{\mathrm{AA}}$ versus centrality in two $p_{\mathrm{T}}$ bins $\left(3<p_{\mathrm{T}}<6.5 \mathrm{GeV} / c\right.$ and $\left.6.5<p_{\mathrm{T}}<30 \mathrm{GeV} / c\right)[22,23]$ (right).

the collective expansion, which is experienced by the charm quarks contained in the fireball [32]. Theoretical models predict in this case a non-zero $\mathrm{v}_{2}$ for the $\mathrm{J} / \psi$ at intermediate $p_{\mathrm{T}}$ [33]. While STAR results for the $\mathrm{v}_{2}$ of the inclusive $\mathrm{J} / \psi$ are compatible with zero everywhere [34], a hint for a nonzero $v_{2}$ can be appreciated at intermediate $p_{\mathrm{T}}$ in the ALICE results $[35,36]$. In Fig. 3 (left), the forward rapidity inclusive $\mathrm{J} / \psi \mathrm{v}_{2}$ is shown as a function of $p_{\mathrm{T}}$ for semi-central collisions (20-60\%) and compared with the predictions of partonic models which indeed reasonably describe the observed behaviour. Depending on the adopted binning for the study, the significance, of the ALICE results for non-zero $\mathrm{v}_{2}$ reaches about $3 \sigma$. This result, complementing the study of the $R_{\mathrm{AA}}$ behaviour, suggests that a significant fraction of the observed $\mathrm{J} / \psi$ is produced from deconfined charm quarks in the QGP phase.

Further insight on charmonium production in $\mathrm{Pb}-\mathrm{Pb}$ collisions can be achieved comparing the higher mass resonances yields, as the $\psi(2 \mathrm{~S})$ one, to the $\mathrm{J} / \psi$. Results, presented as a double ratio of the $\psi(2 \mathrm{~S})$ to $\mathrm{J} / \psi$ yields in $\mathrm{Pb}-\mathrm{Pb}$ and in pp collisions as a function of centrality, are shown in Fig.3 (right) for both ALICE [25] and CMS [38]. CMS observes values higher than 1 in the region $1.6<|y|<2.4$, $3<p_{\mathrm{T}}<30 \mathrm{GeV} / c$, although with rather large normalization errors, mainly due to the low integrated pp luminosity at $\sqrt{s}=2.76 \mathrm{TeV}$. ALICE explores a contiguous range in rapidity $(2.5<y<4)$ and also extend the $p_{\mathrm{T}}$ reach of this measurement down to zero. A significant enhancement in the double ratio for more central collisions is not visible in the ALICE data. However, the large statistics and systematic uncertainties preclude the drawing of strong conclusions on the $\psi(2 S)$ behavior.

At LHC energies, quarkonium study is no more limitied to charmonia, as it was mainly the case at lower energies, but it can be extended also to the bottomonium family. As previously mentioned, the much smaller abundance of bottom quarks with respect to charm quarks implies a negligible contribution from (re)combination processes to bottomonium production. Upsilons are, therefore, a clean probe to get further insight on the balance between regeneration and suppression mechanisms. ALICE [37] and CMS [39] have measured the $\Upsilon(1 \mathrm{~S}) R_{\mathrm{AA}}$ as a function of centrality in two rapidity regions $\left(2.5<y<4\right.$ for ALICE and $|y|<2.4$ for CMS) down to zero $p_{\mathrm{T}}$. As shown in Fig. 4 (left), the observed centrality dependence of the nuclear modification factor is similar for both results, the 

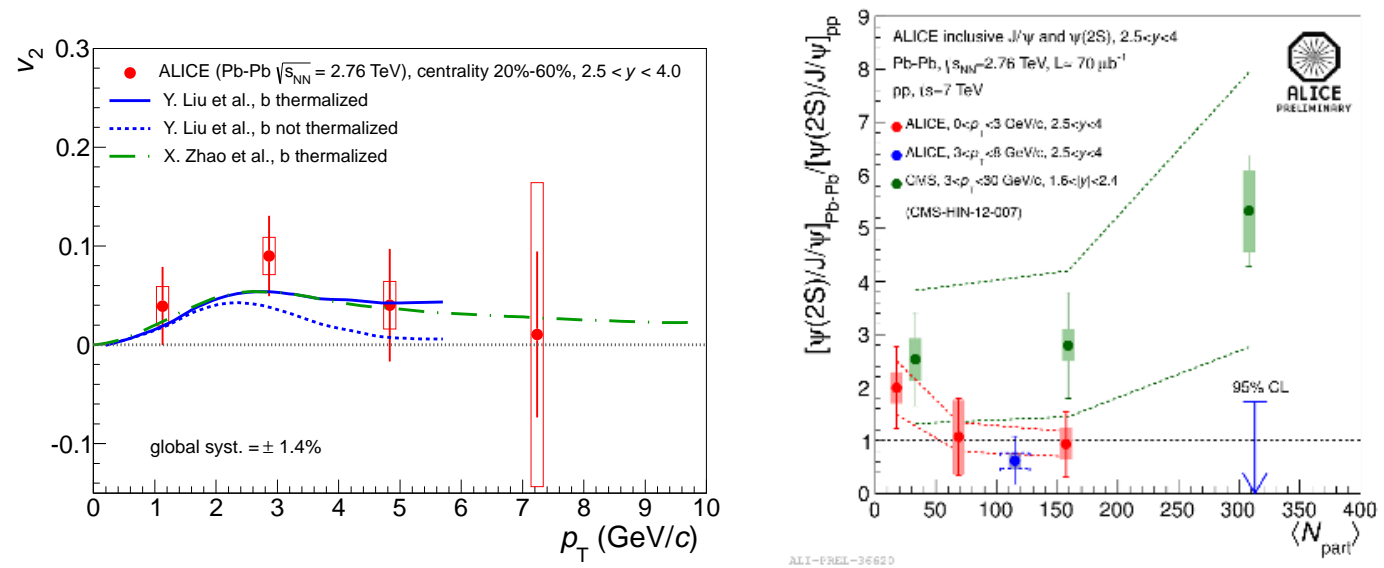

Figure 3. ALICE inclusive $\mathrm{J} / \psi \mathrm{v}_{2}$ versus $p_{\mathrm{T}}$ for semi-central collisions (20-60\%) [35] (left). ALICE and CMS double ratio $\psi(2 \mathrm{~S})$ to $\mathrm{J} / \psi$ in $\mathrm{Pb}-\mathrm{Pb}$ and pp collisions as a function of centrality and in $p_{\mathrm{T}}$ bins $[25,38]$ (right).

suppression being rather constant over the $y$ range covered by ALICE and CMS. The observed amount of $\Upsilon(1 \mathrm{~S})$ suppression might be compatible with the expectations based on the melting of the higher excited states, which contribute, through feed-down, to $\sim 50 \%$ of the observed $\Upsilon(1 \mathrm{~S})$.
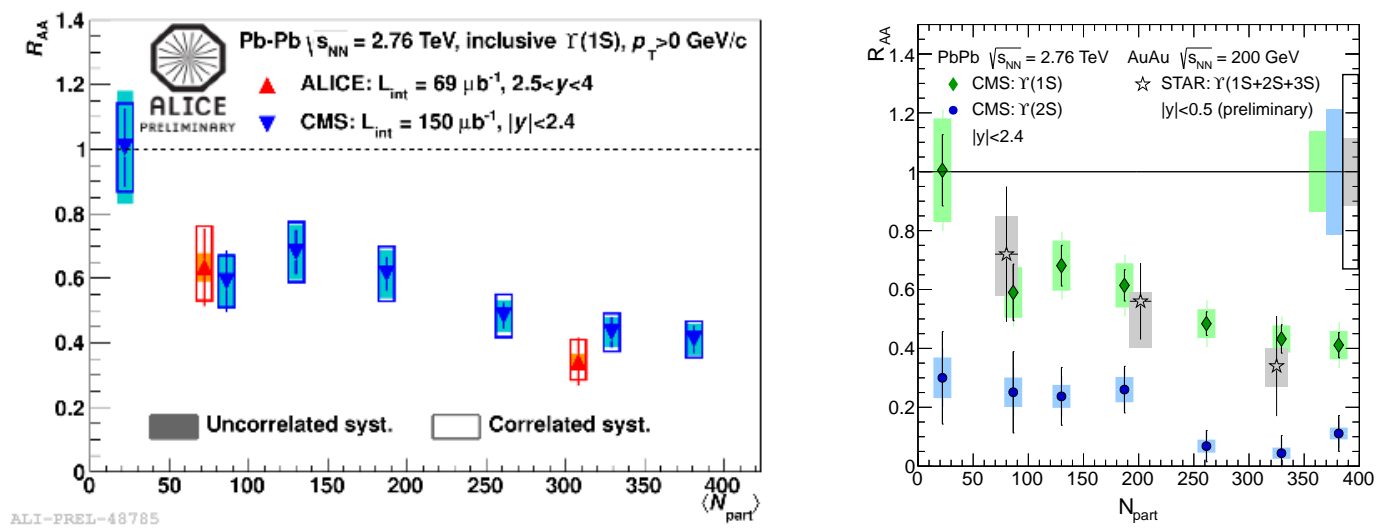

Figure 4. $\Upsilon(1 \mathrm{~S}) R_{\mathrm{AA}}$ measured, as a function of centrality, by the ALICE [37] and the CMS [39] experiments (left). $\Upsilon(1 \mathrm{~S})$ and $\Upsilon(2 \mathrm{~S})$ nuclear modificaton factors measured by CMS, compared to the $R_{\mathrm{AA}}$ of $\Upsilon(1 \mathrm{~S}+2 \mathrm{~S}+3 \mathrm{~S})$ measured by STAR [40] (right). Within large uncertainties, STAR $R_{\mathrm{AA}}$ is compatible with the CMS $R_{\mathrm{AA}}$ for $\Upsilon(1 \mathrm{~S}+2 \mathrm{~S}+3 \mathrm{~S})$.

This hypothesis seems to be confirmed by CMS, being indeed able to measure separately the three $\Upsilon$ states. As shown in Fig. 4 (right) the $\Upsilon(2 S)$ production is, in fact, more strongly suppressed than the $\Upsilon(1 \mathrm{~S})$, already in peripheral collisions. An even stronger $R_{\mathrm{AA}}$ suppression has been measured, centrality integrated, for the $\Upsilon(3 S)$. These results seem to indicate that directly produced $\Upsilon(1 S)$ are not strongly suppressed, while the observed reduction of the yields might be related to the sequential 
suppression of the higher excited $\Upsilon$ states in $\mathrm{Pb}-\mathrm{Pb}$ collisions. However more quantitative conclusions will require precise estimations of the feed-down contributions matching the phase space of the CMS suppression measurement.

Comparing all quarkonium results collected in different kinematic regions, it is clear that in the deconfined medium created in heavy-ion collisions there are two competing mechanisms playing a role, i.e. the suppression by color screening and, for low- $p_{\mathrm{T}}$ charmonia, the (re)combination process. In Fig. 5 the $R_{\mathrm{AA}}$ of high- $p_{\mathrm{T}}$ charmonia and the one of bottomonia, both measured by CMS, is shown as a function of the binding energy of the quarkonium state [23]. In this kinematic region where charmonium (re)combination plays a negligible role, a rather clear ordering of quarkonium suppression might be observed, qualitatively confirming the validity of the sequential melting scenario in heavyion collisions. However, for more quantitative statements, further studies have to be made, to take into account, for example, the effects related to the different kinematic regions in which the various quarkonium states have been measured and the role of higher states feed-down contributions. Furthermore, as previously discussed, to correctly quantify the size of hot matter effects, it is extremely important to estimate cold nuclear matter effects affecting the various quarkonium states.

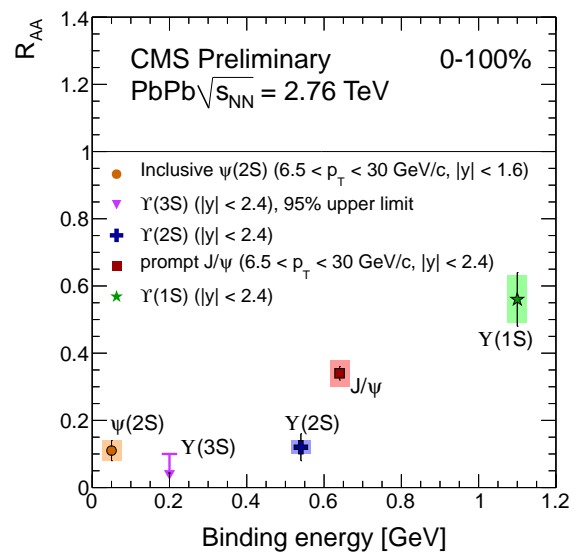

Figure 5. $R_{\mathrm{AA}}$ for several quarkonium states, measured by CMS, as a function of their binding energy [23].

This last issue can now be addressed thanks to the $\mathrm{p}-\mathrm{Pb}$ data recently collected at $\sqrt{s_{N N}}=5.02 \mathrm{TeV}$. Similarly to what is done to quantify how the medium affects quarkonium production in $\mathrm{Pb}-\mathrm{Pb}$ collisions, cold nuclear matter effects are investigated through the nuclear modification factor $R_{\mathrm{pA}}$. Since no pp data at $\sqrt{s}=5.02 \mathrm{TeV}$ were collected, the pp reference is obtained interpolating at $\sqrt{s}=5.02 \mathrm{TeV}$ results collected at higher and lower energies.

First results on the inclusive $\mathrm{J} / \psi R_{\mathrm{pA}}$ have been measured by the ALICE [41] and LHCb [26] experiments. ALICE results in the rapidity ranges $2.03<y_{\text {c.m. }}<3.53$ and $-4.46<y_{\text {c.m. }}<-2.96$ are shown in Fig.6 (left) as a function of rapidity. The positive (negative) rapidity region corresponds to the data collected with the $\mathrm{Pb}$ proton $(\mathrm{Pb})$ beam going towards the ALICE muon spectrometer. While at forward rapidity a suppression of the $\mathrm{J} / \psi$ yield with respect to the pp binary scaled one is observed, no suppression in visible in the backward region. Results are compared with theory predictions, based on a pure nuclear shadowing scenario [42], as well as partonic energy loss, either in addition to shadowing or as the only nuclear effect [43]. Finally, results from a calculation in the CGC framework [44] are also shown. Within the $R_{\mathrm{pA}}$ uncertainties, both the model based on shadowing only and the co- 
herent energy loss approach are able to reasonably describe the data, while the CGC-based prediction overestimates the observed suppression. LHCb has also evaluated the prompt $\mathrm{J} / \psi R_{\mathrm{pA}}$ [26], showing a similar trend as the one observed by ALICE. To avoid the need of the pp reference, another physics observable has been introduced, i.e. the $R_{\mathrm{FB}}$ factor, defined as the ratio of the $\mathrm{J} / \psi$ forward to backward yields. Such a variable has to be studied in the common $y_{\text {c.m. }}$ interval covered both at forward and backward rapidities and it is, therefore, limited to the range $2.96<\left|y_{\text {c.m. }}\right|<3.53 . R_{\mathrm{FB}}$ is studied as a function of $y$ and, as shown in Fig.6 (right), as a function of $p_{\mathrm{T}}$. An increasing trend towards high $p_{\mathrm{T}}$ is observed. Also in this case, as already for the $R_{\mathrm{pA}}$, models based on pure nuclear shadowing or based on coherent energy loss (with or without nuclear shadowing) give a reasonable description of the observed pattern, even if the large theoretical and experimental uncertainties prevent from drawing more precise conclusions on the role of the different contributions.
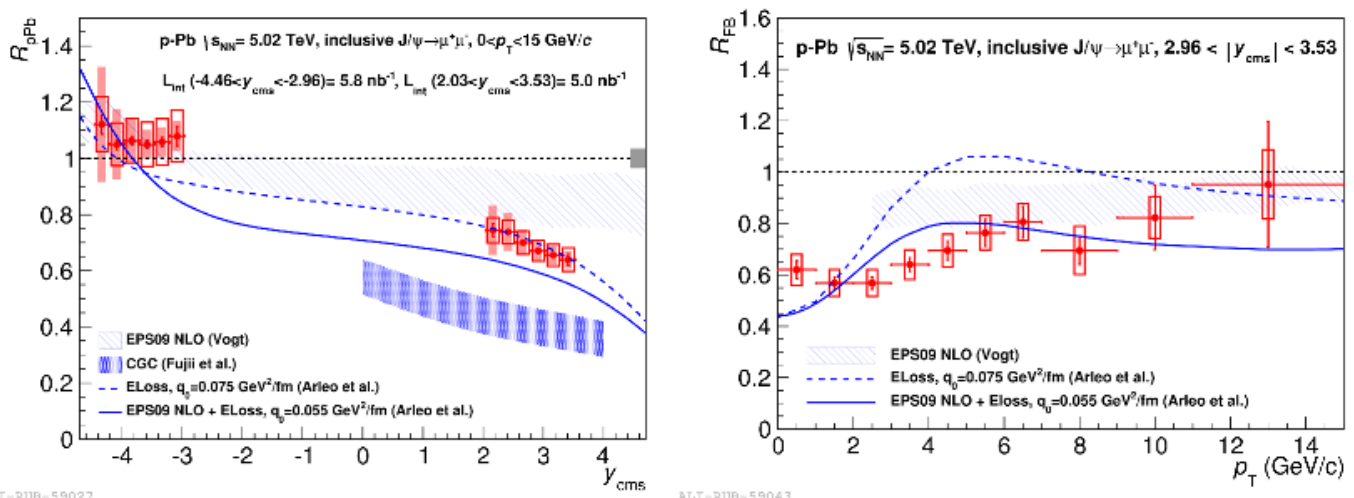

Figure 6. ALICE J/ $/ \psi R_{\mathrm{pA}}$ [41] as a function of $y$ (left) and $R_{\mathrm{FB}}$ as a function of $p_{\mathrm{T}}$ (right), both compared to theory predictions.

\section{Conclusions}

After 25 years since the first studies of quarkonia in heavy-ion collisions, a large wealth of data from the LHC experiments is now available, complementing results from SPS and RHIC. The comparison of several quarkonium states and the different, but complementary, kinematic regions now accessible by the LHC experiments, allows us to get further insight on how the hot created medium is affecting the various resonances. Results have shown that, at LHC energies, there are two competing processes, the suppression in the deconfined medium and the (re)combination of $q$ and $\bar{q}$ states, which have a different role depending on the quarkonium states and on the kinematic region under study. At the same time, other probes, as higher excited states $(\psi(2 \mathrm{~S}), \Upsilon(3 \mathrm{~S}) \ldots)$, or observables as the $\mathrm{J} / \psi$ flow, are still affected by lack of statistics which prevent from drawing firm conclusions on their behaviour. Therefore, we now look forward the future LHC data taking, where higher energies will be reached, allowing to further sharpen our understanding of quarkonium production in the hot deconfined medium.

\section{References}

[1] T.Matsui and H. Satz, Phys. Lett. B178 (1986) 416. 
[2] S. Digal, P. Petreczky and H. Satz, Phys. Rev. D64 (2001) 0940150.

[3] R. L. Thews, M. Schroedter, J. Rafelski, Phys. Rev.C63 (2001) 054905.

[4] P. Braun-Munzinger, J. Stachel, Phys. Lett. B (2000) 196-202.

[5] A. Andronic, P. Braun-Munzinger, K. Redlich, J. Stachel, Journal of Physics G38 (2011) 124081.

[6] A. Aamodt et al. (ALICE Collaboration), Phys. Rev. Lett. 106 (2011) 032301.

[7] K.J. Eskola, H. Paukkunen and C.A. Salgado, JHEP 0904(2009) 065.

[8] D. de Florian, R. Sassot, P. Zurita and M. Stratmann, Phys. Rev. D85(2012) 0704028.

[9] D. de Florian and R. Sassot, Phys. Rev. D69(2004) 0704028.

[10] M. Hirai, S. Kumano and T. H. Nagai, Phys. Rev. C76(2007) 065207.

[11] D. Kharzeev and K. Tuchin, Nucl. Phys. A770(2006) 40.

[12] H. Fujii, F. Gelis and R. Venugopalan, Nucl. Phys. A780(2006) 146.

[13] R. Vogt, Phys. Rev. C61(2000) 035203.

[14] F. Arleo and S. Peigné, Phys. Rev. Lett. 109(2012) 122301.

[15] B. Alessandro et al. (NA50 Collaboration), Eur. Phys. J. C39 (2005) 339.

[16] R. Arnaldi et al. (NA60 Collaboration), Phys. Rev. Lett. 96 (2007) 132302.

[17] A. Adare et al. (PHENIX Collaboration), Phys. Rev. Lett. 98 (2007) 232301.

[18] A. Adare et al. (PHENIX Collaboration), Phys. Rev. C84 (2011) 054912.

[19] N. Brambilla et al., Eur. Phys. J. C71 (2011) 1534.

[20] R. Vogt, Phys. Rev. C81 (2010) 044903.

[21] G. Aad et al. (ATLAS Collaboration), Phys. Lett. B697 (2011) 294.

[22] S. Chatrchyan et al. (ALICE Collaboration), JHEP 05 (2012) 063.

[23] C. Mironov et al. (CMS Collaboration), Nucl. Phys. A904 (2013) 194.

[24] B. Abelev et al. (ALICE Collaboration), Phys. Rev. Lett 109 (2012) 072301.

[25] R. Arnaldi et al. (ALICE Collaboration), Nucl. Phys. A904 (2013) 595.

[26] R. Aaij et al. (LHCb Collaboration) arXiv:1308.6729.

[27] B. Abelev et al. (ALICE Collaboration), Phys. Lett. B718 (2012) 295.

[28] X. Zhao, R. Rapp, Nuclear Physics $\mathbf{A 8 5 9}$ (2011) 114 and priv. comm.

[29] Y.P. Liu et al., Phys. Lett. B678 (2009) 72 and priv. comm.

[30] E. Ferreiro, arXiv:1210.3209

[31] A. Andronic, P. Braun-Munzinger, K. Redlich, J. Stachel, Journal of Physics G38 (2011) 124081.

[32] B. Abelev et al. (ALICE Collaboration), Phys. Rev. Lett. 111 (2013) 102301,

[33] Y. Liu, N. Xu and P. Zhuang, Nucl. Phys. A834 (2010) 317c.

[34] Z. Tang et al. (STAR Collaboration), J. Phys. G38 (2011) 124107.

[35] H. Yang et al. (ALICE Collaboration), Nucl. Phys. A904 (2013) 673.

[36] E. Abbas et al. (ALICE Collaboration), arXiv:1303.5880

[37] L. Manceau et al. (ALICE Collaboration), to appear EPJC, Proceeding of "Large Hadron Collider Physics Conference - LHCP 2013”, Barcelona, 13-18 May 2013

[38] S. Chatrchyan et al. (CMS Collaboration), CMS-HIN-12-007.

[39] S. Chatrchyan et al. (CMS Collaboration), Phys. Rev. Lett. 109 (2012) 222301.

[40] R. Reed et al. (STAR Collaboration), arXiv:1109.3891

[41] B. Abelev et al. (ALICE Collaboration) arXiv:1308.6726.

[42] R. Vogt, arXiv:1301.3395 and priv. comm.

[43] F. Arleo and S. Peigné, arXiv:1212.0434.

[44] H. Fujii and K. Watanabe, arXiv:1304.2221. 